L'article renseigne sur les dernières recherches relatives à la géographie, à l'ethnologie et à l'histoire de l'ile de Pâques.

NUOVE SULL'ISOLA DELLA PASQUA

L'articolo dà informazioni sulle ricerche più recenti fatte sull'Isola della Pasqua.

\title{
PROBLEME DER KARRENBILDUNG
}

\author{
Alfred Bögli
}

\section{EINLEITUNG}

Bei geomorphologischen Untersuchungen in den zentralschweizerischen Kalkalpen fand der Verfasser in den Karrenfeldern eigentümliche Formen, die in ihm Zweifel über das postglaziale Alter der Karren weckten. Daraus entstand das Bedürfnis nach einer neuen Bearbeitung des Karrenproblems.

Die physikalisch-chemischen Arbeitsgrundlagen haben sich seit Jahrzelinten kaum wesentlich verändert, wenn auch an Stelle der alten Auffassung vom Lösungsvorgang des Kalkes die Erkenntnisse über Ionenreaktionen getreten sind. Am Prinzip der Auflösung des Kalkes ändert das nichts. Die Löslichkeit des Kalkes beträgt im kohlensäurefreien Wasser 1,3 Härtegrade $\left(16^{\circ} \mathrm{C}\right)$, beim Partialdruck des Kohlendioxydes von 0,00032 Atmosphären aber 6,5 Grade $\left(15^{\circ} \mathrm{C}\right)$.

Der Einfluß biologischer Vorgänge auf die Korrosionskraft des Wassers ist sehr groß. Nach JeCKLII $^{7}$ reichert sich in der Bodenluft das Kohlendioxyd bis $10 \%$ an. Im Sommer 1947 konnten wir in humusreichen Moränen 8 Volumenprozent nachweisen. Die damit verbundene hohe Lösungsfähigkeit des Bodenwassers bedeutet aber noch keine Förderung der Karrenbildung, da diese im Rahmen der natürlichen Lösungskraft von andern Faktoren bestimmt wird.

D!̣e Pflanzenwelt wirkt vorab durch die Mikroorganismen auf den Kalk ein. Die höheren Pflanzen sind indirekt durch die Schaffung von Humusanreicherungen als dem Lebensraum der Kleinlebewesen daran beteiligt. Bei stauender Nässe entsteht der saure und sehr aggressive Rohhumus, im andern Falle der Edelhumus, der durch die gebildete Kohlensäure auf den Kalk einwirkt. Bei der erhöhten Löslichkeit vermag das Wasser jedoch nicht frei zu fließen und Konvektionsströmungen werden durch die vermodernden Pftanzenteile unterbunden. Die auftretenden Korrosionsformen sind leicht von den Karren zu unterscheiden.

Zahlreiche Autoren haben sich mit den kalklösenden Felsbewohnern beschäftigt. Hier verdienen vor allem die Arbeiten von Blöchliger ${ }^{3}$ und DügGeli ${ }^{4}$ besondere Beachtung. Sie fanden in den Verwitterungsrinden des Schrattenkalkes pro Gramm Oberfächenmaterial Zehntausende von Bakterien, darunter Milch- und Buttersäurebakterien. DügGELI ${ }^{4}$ schreibt mit Recht, es sei festzustellen, inwiefern die nachgewiesenen, steinbewohnenden Spaltpilze sich an den Verwitterungsvorgängen beteiligten, sei es als Produzenten von $\mathrm{CO}_{2}$, sei es als Bildner organischer Säuren oder adsorbtiv ungesättigter Humusstoffe. Die von ihm bei Versuchen beobachtete hohe Korrosion wurde nur dank der Züchtung in den optimalen Verhältnissen einer Nährlösung bei erhöhter Temperatur erreicht. Die Bedingungen auf den Karrenfeldern weichen davon extrem stark ab, so daß die erwähnten Bakterien an der Grenze ihrer Existenzfähigkeit leben. Es fehlt vor allem an genügenden Mengen organischer Substanz, auf die diese heterotrophen Pflanzen angewiesen sind. Erst unter der Humusdecke normalisieren sich die Verhältnisse für die Spaltpilze. Abgesehen vom unbekannten Ausmaße der Korrosionswirkung kann bei der statistisch einigermaßen gleichmäßigen Verteilung der Individuen von einer karrenbildenden oder karrenfördernden Wirkung nicht gesprochen werden.

\section{DER KALKGEHALT DES WASSERS AUS KALKGEBIETEN}

Der Versuch, auf theoretischem Wege die Frage der Kalkauflösung durch die Niederschlagswasser auf eine für die Praxis befriedigende Art zu lösen, findet seine Grenzen in der großen Zahl der Unbekannten. Es ist praktisch auch nicht möglich, sie einzeln zu fassen. Wir beschränkten uns daher darauf, die gesamte Karbonatmenge durch Wasseranalysen zu bestimmen; die Meßergebnisse, das muß betont werden, können gar nichts über die Karrenbildung an sich aussagen, sondern nur das Ausmaß der gelösten Kalkmenge abklären. 
Die Wasserproben wurden durch Titration mit $0,1 \mathrm{n} \mathrm{HCl}$, mit Methylorange als Indikator auf ihre Karbonathärte geprüft. Der Gehalt an freier Kohlensäure wurde mit $0,05 \mathrm{n} \mathrm{NaOH}$ und Phenolphtalein bestimmt.

\section{Das Wasser der Karrenfelder}

Zur Bestimmung der oberflächlich abtransportierten Kalkmengen mußte das Oberflächenwasser vor seinem Verschwinden in den Klüften gefaßt werden.

Das Oberflächenwasser bei Schneeschmelze. Von 1948 bis 1950 wurde an 23 Karren der Kalkgehalt des abfließenden Schneeschmelzwassers bestimmt und in 15 Fällen der Kohlendioxydgehalt gemessen. Die Untersuchungsstellen liegen im Quintnerkalk und erstrecken sich vom Bisistal (850 m) bis zur Glattalp $(1900 \mathrm{~m})$. Die Karren zeigten mit 60 bis $400 \mathrm{~cm}$ normale bis übernormale Längen. Der Kalkgehalt des Wassers lag zwischen 1,3 und 1,6 Härtegraden, was 1,5 bis 2 mg CO2/l voraussetzt. Diese Menge ist zum größten Teile primär im Schneeschmelzwasser enthalten; ein kleiner Teil wurde wahrscheinlich beim Abfließen durch die große Oberfläche aus der Luft aufgenommen. Die Kalkgehalte waren bei verschiedenen Karrenlängen gleich. Freie Kohlensäure konnte keine nachgewiesen werden. Kohlensäurebestimmungen am frischen Schmelzwasser ergaben einen Gehalt von 0,85 bis $1,76 \mathrm{mg} \mathrm{CO} / \mathrm{l}$, was gut mit dem gefundenen Kalkgehalt übereinstimmt. Daraus läßt sich aber auch ableiten, daß die Aufnahme von Luftkohlensäure verglichen mit der Zeit, da das ablaufende Wasser an der Oberfläche verweilt, sehr langsam vor sich geht.

Schmelzwasser, das aus Humuspolstern abtropfte, wies Härtegrade von 2,8 bis 4,6 auf bei einem Gehalt an freier, aggressiver Kohlensäure bis zu 2,6 mg. Da an diesem Tage die Schmelze kurz vor der Messung eingesetzt hatte, müssen diese Werte infolge Vermischung mit älterem, kalkreichem Wasser Maximalwerte sein.

In mehreren Grabungen wurde auf Glattalp $(1800 \mathrm{~m})$ versucht, Tropfwasser direkt unter der Schneedecke zu fassen. Es konnte jedoch trotz der seit einigen Tagen erfolgenden Schneeschmelze kein subnivales, rinnendes Schmelzwasser festgestellt werden. Nach brieflicher Mitteilung des LawinenInstitutes Davos-Weißfluhjoch hielt sich die Obergrenze des subnivalen Schmelzwassers in den Alpen im Winter 1947/48 durchschnittlich auf ca. $1800 \mathrm{~m}$ Höhe. Diese Grenze quert die zentralschweizerischen Karrenfelder; die obersten Teile werden auch in warmen Wintern nie darunter liegen, die tiefern wohl immer darunter bleiben.

Von einem stärkeren Einfluß des winterlichen Schneeschmelzwassers kann entgegen der bisherigen Auffassung nicht gesprochen werden.

Das Oberfächenwasser der Karren bei Regen. Vom 10. bis 13. August 1948 wurden auf Glattalp und Karrenalp 15 Untersuchungen über den Kalkgehalt des über die Karren abfließenden Wassers durchgeführt. Sie ergaben beim Fehlen von Humuspolstern Werte zwischen 1,5 und 2,7 Härtegraden, während bei deren Anwesenheit der Gehalt auf 3 Härtegrade stieg.

Bei Beginn eines Regens wurden auf Glattalp $(1850 \mathrm{~m})$ 2,7 Härtegrade gemessen, bei voller Entwicklung des Niederschlages 1,5 Härtegrade. Nach Aufhören des Regens stieg der Kalkgehalt im Restwasser auf 1,7 Härtegrade. Auf der Karrenalp (1950 m) erreichte der Kalkgehalt nach den ersten $5 \mathrm{~m}$ einer extremen langen Rinnenkarre 2 Härtegrade. Nach $10 \mathrm{~m}$ enthielt das Wasser, das vorher durch ein Humuspolster von $4 \mathrm{~m}$ Länge geflossen war, vor dem Verschwinden in die Kluftkarre 3 Härtegrade. Diesem Wasser war noch das vom Polster zurückgehaltene Wasser eines früheren Niederschlages beigemischt.

Ein kleiner Korrosionskolk auf Glattalp endete einseitig an einem Humuspolster. Sein Wasser enthielt 8,5 Grade Kalk, also zwei Grade mehr als dem Partialdruck der Luftkohlensäure entsprochen hätte. Das Wasser des Tümpels (ca. 3 I Inhalt) hatte mehrere Tage Zeit, das Kalk-Luftkolılensäuregleichgewicht zu erreichen. Das läßt Rückschlüsse auf die Entstehung des Kalkgehaltes der oben erwähnten großen Karre zu. In ihrer Nähe zeigte eine humusfreie $9 \mathrm{~m}$ lange Rinnenkarre nur 1,9 Härtegrade Kalk.

Als Vergleich zu den bisher angeführten Karren muß festgestellt werden, daß Rinnenkarren im Untersuchungsgebiet nur selten $3 \mathrm{~m}$ Länge überschreiten.

Karrenwasser aus Felsspalten und Sickerstellen. Quellen und Sickerstellen sind in den Karrenfeldern selten, besonders wenn man jene Fälle abtrennt, die irdendwie aus tonigen Relikten, Aufschüttungen oder Moränen stammen. Wo das Wasser aus humusarmen Karrengebieten kommt, erreicht es 7 Grad Härte, entsprechend dem Kohlendioxydpartialdruck der Luft. Die Quellen aus Karren mit zahlreichen Humuspolstern weisen einen Kalkgehalt von 8,5 Graden auf, was die Anwesenheit biologischer Kohlensäure beweist.

\section{Folgerungen}

Der Kalkgehalt des Wassers, das in den Klüften verschwindet, hebt sich nicht wesentlich über die Löslichkeit des Kalkes in kohlensäurefreiem Wasser hinaus. Das beweist, daß das Gleichgewicht zwischen dem Kalk und dem Wasser mit dem 
darin gelösten Kohlendioxyd sich zwar recht schnell einstellt, daß aber jenes zwischen dem Wasser und dem Kohlendioxyd der Luft erst nach geraumer Zeit erreicht wird. Es ist daher nicht wesentlich, ob in einem bestimmten Zeitraum bei Nebelregen wenig, oder bei Platzregen viel Wasser über den Kalk ablaufe. In beiden Fällen werden ähnliche Kalkmengen aufgelöst werden, was durch die verschiedenen Gehalte des abfließenden Wassers nachgewiesen werden konnte. Die Proportionalität zwischen dem abtransportierten Kalk und der Niederschlagsdauer ist daher strenger als jene zwischen ihm und der Niederschlagsmenge. Trotzdem wird man sich an letztere halten müssen, da über die Niederschlagsdauer, genauer über die Zeit, während welcher Wasser über den Kalk fließt, noch kein Material veröffentlicht wurde.

In der Hauptzone der Karrenfelder $(1500 \mathrm{~m}$ bis $2400 \mathrm{~m})$ steigt der Anteil des Schnees an den Niederschlägen von $40 \%$ auf $67 \%$. Die geringe Wirkung des Schneewassers zeigt aber, daß an der Auffassung von einer vorwiegend subnivalen Entstehung der Karrenfelder nicht mehr festgehalten werden kann. Daß aber einzelne Karren durch die Schneedecke wesentlich in ihrer Entwicklung gefördert werden, kann man immer wieder beobachten.

Zusammenfassend darf angenommen werden, daß das Jahresmittel der oberflächlich weggeführten Kalkmengen bei 1,7 Härtegraden liegt.

Die Muota führt aus ihrem Einzugsgebiet in Jahıresdurchschnitt der teilweise sehr nassen 15 Jahre von 1932 bis 1946 ungefähr $1880 \mathrm{~mm}$ Niederschläge ab. Da die Karrenfelder im regenreichen Teile liegen, so darf der versickernde Anteil mit etwa $2000 \mathrm{~mm}$ angenommen werden, nach O. LürschG 13 einem durchschnittlichen Niederschlage von $22^{\prime} 75 \mathrm{~mm}$ entsprechend. Die pro Quadratmeter abfließenden 2000 Liter Wasser führen bei der Annahme eines Kalkgehaltes von 1,7 Härtegraden $34 \mathrm{~g}$ Kalk, entsprechend 12,5 ccm Gestein davon. Das sind jährlich 0,00125 $\mathrm{cm}$ Abtrag. Die wenigen Prozente an Beimengungen von Ton beeinflussen diesen Wert nicht wesentlich.

Einige Schwierigkeiten bereitet die Bestimmung des Gesamtabtrages auf der Oberfläche seit dem Eisfreiwerden, da die Autoren verschiedene Jahreszahlen angeben.

\begin{tabular}{lrrrrr} 
Autor & Jahr & Lit. & \multicolumn{1}{c}{ Daun } & Gschnitz & Bühl \\
\hline WeLten & 1944 & 16 & $7700 \mathrm{~J}$. & $8000 \mathrm{~J}$. & $10000 \mathrm{~J}$. \\
KAYSER & 1948 & 8 & $10000 \mathrm{~J}$. & $10500 \mathrm{~J}$. & $14000 \mathrm{~J}$.
\end{tabular}

Die Jahreszahlen von WelteN sind auf der Pollenanalyse aufgebaut. Ihre Grundlage besteht in der Auszählung warvenartiger Schichten im Faulenseemoos bei Spiez, unter starker Berücksichtigung der außeralpinen Vorkommen. Die Lage dieses Mooses innerhalb des Alpentores ermöglichte dem Autoren eine Parallelisierung mit den inneralpinen Rückzugsstadien der Gletscher.

Die Schneegrenzendepression betrug zur Daunzeit $300 \mathrm{~m}$, zur Gschnitzzeit $600 \mathrm{~m}$. Der Zeitraum zwischen Gschnitz und Bühl ist in der Schweiz in dieser Hinsicht nicht untersucht worden. In diese Zeit fällt das Schlernstadium, das wohl näher an Gschnitz liegt, denn seine Gletscher stießen vor, als die Haupttalgletscher schon verschwunden waren. Es wurde bisher in der Schweiz nicht beobachtet. Seine Schneegrenzendepression beträgt 800 bis $900 \mathrm{~m}$ (Lit. 9). Die heutige Schneegrenze liegt im Gebiet der Muotataleralpen bei $2500 \mathrm{~m}$, so daß für Gschnitz eine Firnbedeckung der Karrenfelder angenommen werden darf. Der gesamte korrosive Abtrag seit Gschnitz berechnet sich je nach den eingesetzten Jahreszahlen, auf $10 \mathrm{~cm}$ bezw. $13 \mathrm{~cm}$.

Seit dem Gschnitzstadium hat sich das Klima mehrfach geändert. Zur Bronzezeit lag die Schneegrenze vermutlich sogar $300 \mathrm{~m}$ höher als heute (Lit. 8, S. 296), 
wahrend im 17. Jahrhundert mit einer Depression von $50 \mathrm{~m}$ gerechnet werden muß. Wir leben wahrscheinlich in einem verglcichsweise feuchten Klima, so daß die Abtragswerte, bezogen auf das Mittel der Nंiederschläge seit Gschnitz, eher zu hoch berechnet wurden.

\section{Das Wasser der Fels- und Stromquellen}

Fels- und Stromquellen sind in ihrem Wesen nahe verwandt und unterscheiden sich nur durch ihre Wasserführung.

Felsquellen. Es konnten an 13 Felsquellen Messungen durchgeführt werden. Jene, die mit grösseren Karrengebieten zusammenhängen, zeigen einen Durchschnitt von 8,3 Härtegraden, jene aus stark bewachsenen Gebieten mit tonigen Böden einen solchen von 13 Härtegraden. Das Wasser wandert in diesen so langsam durch die humose Bodenschicht, daß ein Ausgleich zwischen dem Kohlendioxyd der Bodenluft und der Kalklösung sich einstellen kann.

Stromquellen. Stromquellen sind eine Karsterscheinung und stehen damit den Quellen und Sikkerwasserstellen in Karrenfeldern nahe. Sie sind im Muotatal und Bisistal häufig. An ihnen läßt sich der Wechsel des Kalkgehaltes mit der Wasserführung leicht beobachten. Der „schleichende Brunnen “ besaß bei Niedrigwasser vor der Schneeschmelze 10,5 Härtegrade Kalk, bei Hochwasser dagegen nur 8,5. Diese Änderung ist u. E. nicht so sehr eine Folge mangelnden Kontaktes mit den Kluftwänden, als eine Folge des Zeitmangels für das Erreichen des Gleichgewichtes zwischen der Kohlensäure der Bodenluft und der Kalklösung.

Am Fuße der Abstürze der Fronalp gegen das Muotatal kommen mehrere Stromquellen zum Vorschein, deren Einzugsgebiet überwiegend tonig-lehmige Gesteine mit sauren Böden aufweist. Entsprechend liegt ihr Kalkgehalt bei 12 Härtegraden.

\section{Verschiedene Wasseraustritte}

Bei den Schuttquellen des Muotatales dürfte es sich meist um verdeckte Felsquellen handeln. Mit steigendem Tongehalt des Schuttes steigt auch der Kalkgehalt des Wassers. Vier Quellen zeigten Werte von 9,4 bis 10 Härtegraden Kalk, was auf verdeckte Felsquellen hinweist. Das Mittel der übrigen 23 Messungen ergab 12,1 Härtegrade.

Von weitern 17 Messungen ergaben die Grundwasseraufstöße bei Ried (Muotatal) Kalkgehalte von 14 bis 14,2 Graden, während einige Brunnen, deren Wasser aus lehmigen Böden stammt, noch mehr enthielten. Hier wurde das Maximum mit 17,5 Graden angetroffen.

6 Messungen wurden an Höhlenwasser im Lauiloch unterhalb Illgau durchgeführt. 250 bis $500 \mathrm{~m}$ höher liegen über Tag Wälder, Wiesen und Weiden. Der Kalkgehalt schwankt zwischen 10,5 und 14 Härtegraden.

\section{Zusammenfassung}

Die Quellen des Muotatales zeigen eine deutliche Abhängigkeit ihres Kalkgehaltes von den Böden ihrer Einzugsgebiete. Reine Kalke lassen das Wasser rasch aus der Zone starker biologischer Vorgänge in die Tiefe verschwinden, so daß die Quellen einen niedrigen Kalkgehalt unter 10 Graden aufweisen. Tonige Böden verlangsamen die Wanderung des Wassers derart, daß ein Gleichgewicht zwischen dem Kohlendioxyd des Bodens und der Kalklösung sich einstellen kann. Entsprechend ist der Kalkgehalt dieser Quellen höher.

\section{MORPHOGRAPHIE DER KARREN}

Die Bildung der Karren ist eine Folge der Wechselbeziehungen zwischen löslichem Gestein, Atmosphäre, Orographie und Hydrographie. Niederschlagswasser und Gestein sind die Faktoren erster Ordnung, das Wasser als aktiv lösendes Agens, das Gestein passiv als lösbares Medium.

Die Niederschläge können als solche an der Karrenbildung nicht beteiligt sein, wirken doch die Hagelkörner und Regentropfen im allgemeinen diffus auf die Kalkflächen ein, da die Aufschlagstellen statistisch ziemlich gleichmäßig über das Feld verteilt sind. Erst das abfließende Niederschlagswasser, stamme es nun vom Regen oder von schmelzendem Hagel oder Schnee, vermag Karren zu bilden. Es wirkt vor allem lösend, jedoch auch mechanisch. Bei der gleichsinnigen Wirkung beider kann experimentell oder am natürlichen Objekt keine Entscheidung über das Aus- 


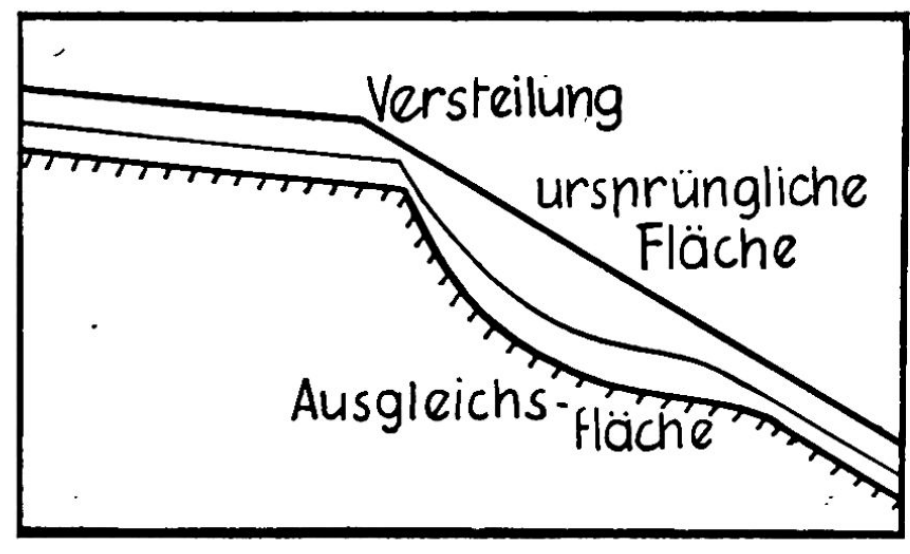

Abb. 1

maß getroffen werden. Doch ist bei der großen Berührungsfläche zwischen Gestein und abfließendem Wasser die Fließgeschwindigkeit eher klein und damit auch die kinetische Energie, die Ursache der Erosion. Dazu kommt der meist innige Verband der Kalkkörner, der ein Losreißen sehr erschwert. Die mechanische Wirkung ist daher unbedeutend.

Das Ausmaß der oberflächlichen Kalklösung ist beschränkt. Während das Gleichgewicht zwischen Kalk und Wasser sich schnell einstellt, verläuft die Kohlensäureaufnahme aus der Luft so langsam, $\mathrm{da} ß$ das Wasser auf der kurzen oberirdischen Laufstrecke nur geringe zusätzliche Kalkmengen zu lösen vermag. Daher bleibt der Kalkgehalt meist unter 2 Härtegraden. Das Gleichgewicht zwischen dem festen Kalk, dem gelösten Calciumkarbonat und dem gelösten, sog. freien Kohlendioxyd kann im Wasser nur erreicht werden, wenn die zum gelösten Kalke zugehörige freie Kohlensäure dem Partialdruck der Luftkohlensäure entspricht. Dieser Wert ist stark von der Temperatur abhängig und liegt zwischen 1 und $1,5 \mathrm{mg} \mathrm{CO} / 1$, entsprechend einer Härte von 6,8 bis 8 Graden. Erst die Wanderung durch die Klüfte dauert lange genug, daß sich das Gleichgewicht einstellen kann.

Die Kalkkonzentration des Wassers nimmt beim Abfließen über die Kalkfläche zu; seine Korrosionsfähigkeit sinkt. Es ist evident, daß auf einer solchen Grundlage niemals Karrenfelder entständen, müßten doch die Karren nach unten verflachen. Dies widerspricht allen Beobachtungen. Nur die flächenhafte Benetzung durch korrosionsfähiges Wasser vermag Karren zu bilden, da nur dann die Selbstabschwächung aufgehoben ist.

Kalk ist an sich wasserundurchläßig. Infolge seiner Sprödigkeit ist er aber meist zerklüftet. Die Klüfte sind die gegebenen Orte der Ableitung der Oberflächenwasser in den Untergrund und geben Anlaß zur Bildung von Kluftkarren, Karrenschloten, Karrenbrunnen, Dolinen und Schlundlöchern.

Die Schichtung des Kalkes ist für die Bildung der Karrenformen bedeutungsvoll. Schon ein geringfügiger Wechsel im Chemismus genügt als Ansatz zur Modifikation der Lösungswirkung. Aber nur in seltenen Fällen entstehen Komplexe einheitlicher, von den übrigen abweichender Formen.

Die Bankung des Gesteins ist für die Erhaltung der Karren wichtig. Bei dünnbankigen Kalken werden die Bänke durch die Karren durchschnitten. Die Schichtfläche leitet das Wasser leicht, so daß auch von hier aus der $Z$ usammenhang gelockert wird. Das Gestein zefällt im Verlaufe der Karrenentwicklung bald. Das entstehende Trümmerfeld läßt sich nur schwer als ein Produkt der Verkarstung bestimmen (s. Trümmerkarren S. 201).

Die Struktur und vor allem der Chemismus sind für die Bildung und Erhaltung der Karrenformen ausschlaggebend. Dichter und reiner Kalk gibt die schönsten und widerstandsfähigsten Karren. Mit zunehmender Korngröße steigt die Wirkung des Spaltenfrostes. Auf grobkörnigen Kalken, z. B. Echinodermenbreccien, Nummelitenkalk und Sandkalk bilden sich zwar schöne Karren, doch sind deren Kanten gerundet. Bei zunehmendem Tongehalt laufen die karrenbildenden Vorgänge, abgesehen von der Selbstabschwächung durch die tonigen Rückstände, auch weiterhin ab, doch werden die dabei entstehenden Formen immer schneller durch die Verwitterung zerstört; die Karren werden runder und flacher.

Die Verwitterungsrinde des Kalkes saugt infolge ihrer Porosität Wasser auf und hält es kapillar und adhäsiv fest. Die dabei entstehende gesättigte Kalklösung bildet eine Trennungsschicht $z$ wischen dem Kalk und dem abfließenden Niederschlagswasser. Dieses ungesättigte Wasser wird immer Teile der Grenzschicht wegreißen. Wo das Gefälle plötzlich zunimmt, verringert sich wegen des Beharrungsvermögens des fließenden Wassers dessen Wirkung auf die Grenzschicht, was gleich- 


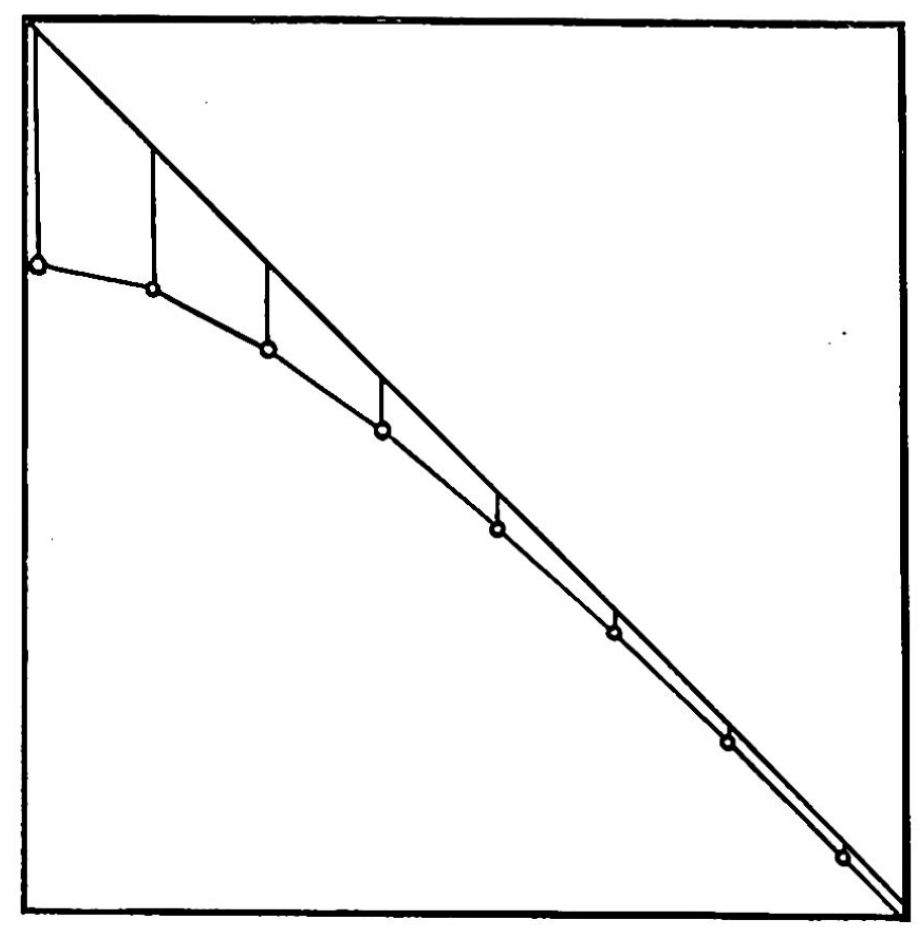

Abb. 2

bedeutend ist mit verringerter Korrosion. Unter der Wirkung der Gravitation biegt sich dann die Bewegungsrichtung nach unten und weist in spitzem Winkel auf die Kalkfläche. Zur schwerkraftbedingten Druckkomponente kommt noch die Druckkomponente des aus der Richtung gedrängten Wassers und seine höhere Fließgeschwindigkeit. Das bedeutet erhöhte Wirkung auf die Grenzschicht, somit erhöhte Korrosion. In der Versteilung folgt somit auf eine Zone verringerter Lösung eine solche der verstärkten; die Steile wird steiler (Abb. 1). Dies wird noch prägnanter, wenn wieder eine flachere Stelle folgt. Es entsteht eine Hohlform, deren Boden sich allmählich der Waagrechten nähert. Der Prozeß kommt erst zum Stillstand, wenn durch den Rückstau des verlangsamt weiterfließenden Wassers die Bewegungsenergie aufgebraucht wird. Solche Flächen werden im weitern als Ausgleichsflächen bezeichnet. Sie spielen bei vielen Karrenformen, vor allem bei Trichterkarren, eine bedeutende Rolle.

Aus thermodynamischen Gründen ist zu erwarten, daß im Idealfall pro Zeitintervall immer der gleiche Anteil der noch vorhandenen Lösungsfähigkeit ausgenützt wird. Um die Verhältnisse auf einer schiefen Kalkplatte überblicken zu können, zerlegen wir den Vorgang der Auflösung und integrieren hierauf. Wir nehmen an, Regenwasser tropfe nur entlang einer waagrechten Linie auf eine homogene, geneigte Kalkfläche und laufe mit einigermaßen konstanter Geschwindigkeit darüber hinunter. Die pro Zeitintervall gelöste Kalkmenge nimmt in geometrischer Reihe $a b$ und nähert sich asymptotisch dem Werte Null. Oben entsteht infolgedessen eine Verflachung, die nach unten allmählich in die urspüngliche Fläche übergeht (Abb. 2). Eine zweite, tiefere Tropfenreihe wirkt wie die erste, sich zu jener summierend. Durch Addition weiterer Tropfenreihen, entsprechend einer flächenhaften Benetzung durch Regen, entsteht eine neue Form, die von der ersten grundlegend abweicht. Statt der Verflachung bildet sich oben eine Versteilung, die nach unten flacher wird und zuletzt subparallel zur alten Oberfläche verläuft (Abb. 3. 《unkorrigierte Kurve »). Diese Grundform untersteht mannigfachen Veränderungen. In einigem Abstand von der Ablaufkante erreicht die Wirkung des abfließenden Wassers ein Maximum, weil hier zur relativ starken Richtungsänderung mit 


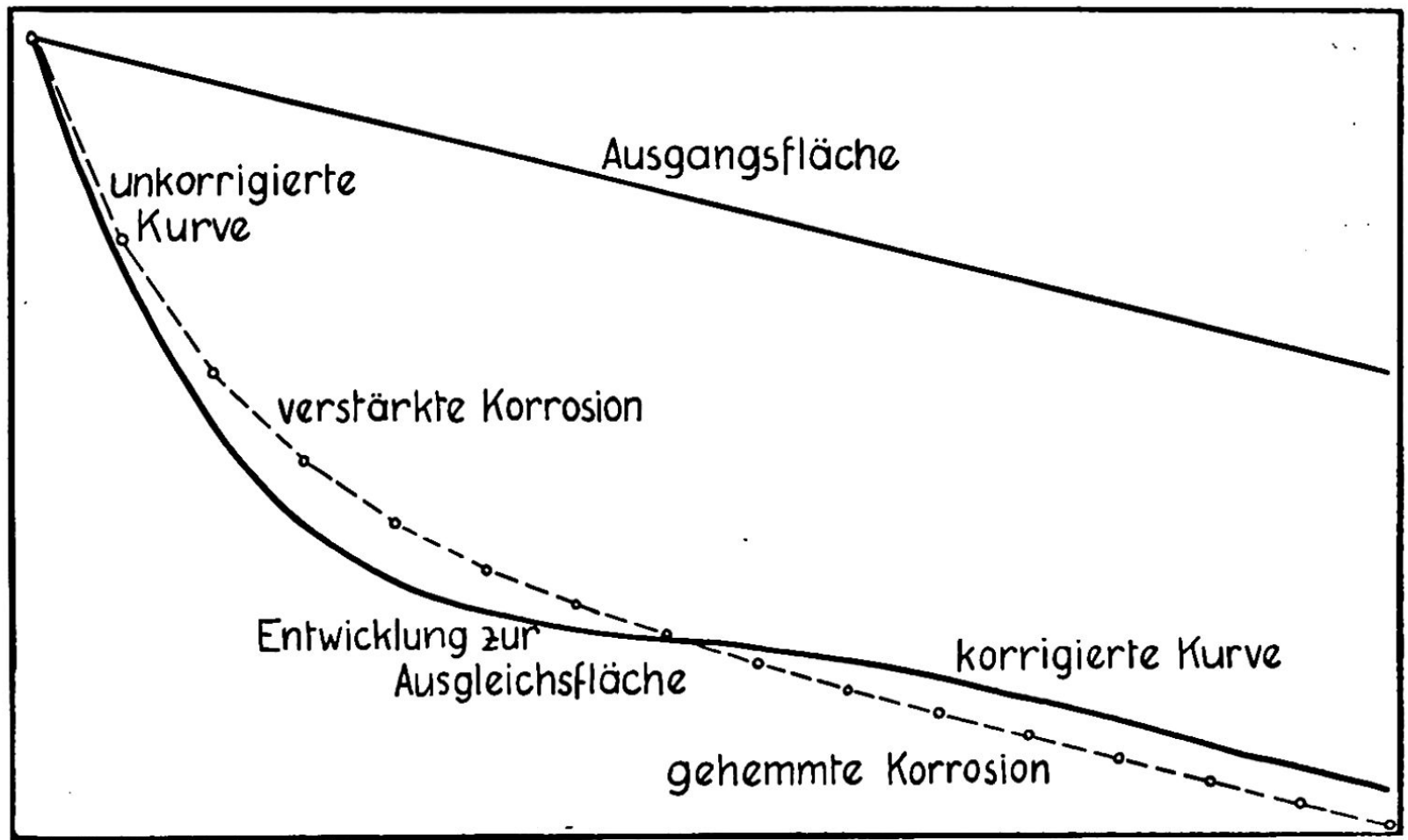

Abb. 3

entsprechendem Einfluß auf die Grenzschicht noch die größere Wassermenge mit erhöhter Fließgeschwindigkeit kommt. Dies addiert sich zur bisherigen Form, so daß eine zusätzliche Vertiefung eintritt. Weiter unten bildet das abfließende Wasser ein Auffangpolster für das lösungskräftige neue Regenwasser, das so vom Gestein ferngehalten wird und sich nur allmählich mit dem übrigen Wasser vermischt. Die Lösung erreicht hier somit nicht das theoretisch verlangte Ausmaß. So bildet sich am Fuße der obersten Versteilung allmählich eine Hohlform, die auf einen Ausgleichsboden ausmündet (Abb. 3, «Ausgleichsfläche »). Diese Kurve ist die Grundform der primären Karrenbildungen.

Die Bildung der Karren und damit der Karrenfelder setzt nach Obigem frei fließendes, aus flächenhafter Benetzung herstammendes Wasser voraus. Diese Bedingung wird in größerem Ausmaße nur durch Nacktheit, also Vegetationslosigkeit der Kalkflächen erfüllt. Nackte Flächen können durch Kälte oder immer wiederkehrende Trockenzeiten nackt bleiben. Deshalb sind auf der Alpennordseite die höheren Lagen bevorzugt, während im Süden das Mittelmeerklima die Bildung von Karren in geringen Höhen fördert. Doch findet man bei Muotatal Karren felderweise bis zum 'Talboden auf $650 \mathrm{~m}$. Auf dem Gibel ob Schwyz reicht das bewachsene Karrenfeld bis $700 \mathrm{~m}$ hinunter. Dies spricht für ein ehemals anderes, kühleres Klima, das die Entstehung der Karrenfelder begünstigte. Große Quintnerkalkblöcke unterhalb Bisistal $(850 \mathrm{~m})$ zeigen sowohl schöne Rinnen- als auch Rillenkarren, da an ihnen die Voraussetzung der Vegetationslosigkeit auch heute noch erfüllt ist.

Die Einteilung der Karren nach morphographischen und genetischen Gesichtspunkten ergibt drei Grundformen, die sich weitgehend variieren lassen.

\section{Kluftkarren. \\ 2. Rinnenkarren s. $l$. \\ 3. Rillenkarren (Kannellierungen).}

Kluftkarren bilden die bestdefinierte Karrenform. Sie entstehen durch korrosive Erweiterung von Klüften. 
Nach LeHmanN ${ }^{11}$ vermögen auch Klüfte kapillaren Durchmessers wegsam zu werden, wenn das kapillar festgehaltene Wasser unter Druck gesetzt wird. Es kommt zum Wandern; dabei neigt es zur Bevorzugung einzelner Wanderwege, was zur Entstehung von Karrenröhren und Karrenschloten führen kann. Eine Trennung zwischen den tektonischen Hauptklüften und dem Mohrschen Scheerflächensystem einerseits und den morphologisch bedingten Klüften, erzeugt durch die randlichen Abstürze andererseits, konnte durch die Kluftkarrenanalyse nicht erreicht werden, obschon ohne Zweifel Torsionsbrüche im Sinne der Daubreeschen Versuche (Lit. 11) vorhanden sind.

Rinnenkarren s. $l$. sind Korrosionsgebilde verschiedener Form, die durch das Abfließen des Niederschlagswassers über eine Kalkfläche entstehen. Mit Ausnahme der Abhängigkeit vom Gefälle scheint ihnen jede äußere Gesetzmäßigkeit zu fehlen. Das Wasser verschwindet im Gegensatz zu den Kluftkarren nicht im Gestein. Rinnenkarren enden daher entweder an den Kluftkarren oder münden auf undurchlässige Böden oder Gesteine aus. Der bei geringem Gefälle große Einfluß des Gesteins nimmt mit zunehmender Neigung $a b$ bis zur Bedeutungslosigkeit.

Rinnenkarren s. s. gleichen fluvialen Formen.

Dies scheint ein Widerspruch zu sein, folgt doch die Korrosion den Lösungsgesetzen. Der Träger der Korrosion ist aber das Wasser, das den hydrodynamischen Bewegungsgesetzen unterworfen ist und dadurch sowohl den Ort größter Korrosion als auch Erosion bestimmt. Der Seitenerosion entspricht die Seitenkorrosion, der Tiefenerosion die Tiefenkorrosion, die allerdings nur geringe Abhängigkeit vom Gefälle zeigt. Steilen wandern rückwärts, verstärken sich und werden zuletzt überhängend. Korrosionskolke können sich beliebig eintiefen, da keine festen Bestandteile weggeschwemmt werden müssen. Trotzdem sind tiefe Korrosionskolke selten, denn der Ersatz des gesättigten Wassers wird mit zunehmender Tiefe immer unvollständiger. Solche Kolke können Anlaß zur Ansiedlung von Pflanzen geben, die mit Hilfe der biologisch entstandenen Kohlensäure zuerst den Eintiefungsvorgang beschleunigen, dann aber infolge Verhinderung der Wasserzirkulation durch die Humusanhäufung unterbinden. Wie stark bei der Bildung dieser Hohlformen die Humuslager beteiligt sind, ist nicht $\mathrm{zu}$ entscheiden.

Bei kleinerem Gefälle winden sich die Rinnenkarren, stark beeinflußt durch die Inhomogenitäten des Gesteins, in eingetieften Mäandern, da der "Vorfluter “ eine tiefe Korrosionsbasis darstellt. Nicht selten liegt in der eigentlichen Karrenrinne noch ein Niedrigwasserbett. Das zeigt, daß die korrodierende Wirkung des Niederschlagswassers Karren bildet, während das Schneeschmelawasser lokaler Herkunft auf seinem Wege in der Tiefe der Karre ein Gerinne erzeugt, ähnlich den Korrosionsrinnen in Höhlen. Im Prinzip müßten solche Rinnen mangels Zufuhr korrosionsfähigen Niederschlagswassers durch Erreichen des Gleichgewichtes verflachen und zuletzt ganz verschwinden. Rinnen von solcher Länge konnten wir bisher nicht finden. Anders liegt der Fall da, wo im Schatten einer Steilstufe liegende Schneemengen weit in den Sommer hinein Schmelzwasser auf annähernd flach liegende Kalkflächen abgeben. Hier können gewundene Korrosionsrinnen ihren Ausgang nehmen. Sie verflachen allmählich und lösen sich auf, da beim Weiterfließen auf der sonnenbeschienenen Fläche das Wasser verdunstet (Märenberge $2300 \mathrm{~m}$ ).

Der Querschnitt der Rinnenkarren ist typisch. Von der Karrenschulter bricht die Fläche in mehr oder weniger scharfer Kante ab zur Senkrechten. Der Karrenboden ist meist gerundet, seltener flach oder anders geformt. Das Wasser, das auf der Schulter fließt, ist dank der dauernden Zufuhr aus den Niederschlägen korrosionsfähig. Die Korrosion wird beim Übergang zur Senkrechten fast Null. In der Tiefe, an der Umbiegung zum Karrenboden wird die Grenzschicht, die unter der größeren und schneller bewegten Wassermenge ohnehin schon reduziert wurde, dadurch verstärkt angegriffen. Die Karre vertieft sich ohne wesentliche Breitenzunahme. Der Einfluß des bei Schneebedeckung direkt hineintropfenden Schmelzwassers darf nicht unterschätzt werden.

Je steiler das Gelände ist, um so mehr streckt sich der Lauf des Wassers, um so weniger wirken die physikalischen und chemischen Inhomogenitäten des Gesteins. Zuletzt fließt das Wasser geradlinig in der Richtung des Gefälls, parallele Regenrinnen bildend.

Der Begriff der Regenrinnen wird auch bei der Denudation im Sinne von Rachel und Spülrinne verwendet. Da er aber nicht festgelegt ist und durch die beiden andern Ausdrücke ersetzt werden kann, so stehen wir nicht an, das Wort im Sinne einer Karrenform zu verwenden, wo sich dies ohne Gefahr der Verwechslung tun läßt.

Eine eigentümliche Karrenform, die wir als Trichterkarren bezeichnen, wurde oberhalb $1900 \mathrm{~m}$ im Bereiche langandauernder, hoher Schneebedeckung angetroffen (Abb. 4). Sie besteht aus einem Ausgleichsboden, der halbkreisförmig von einem Wändchen von wenig Zentimetern Höhe umgeben ist. 


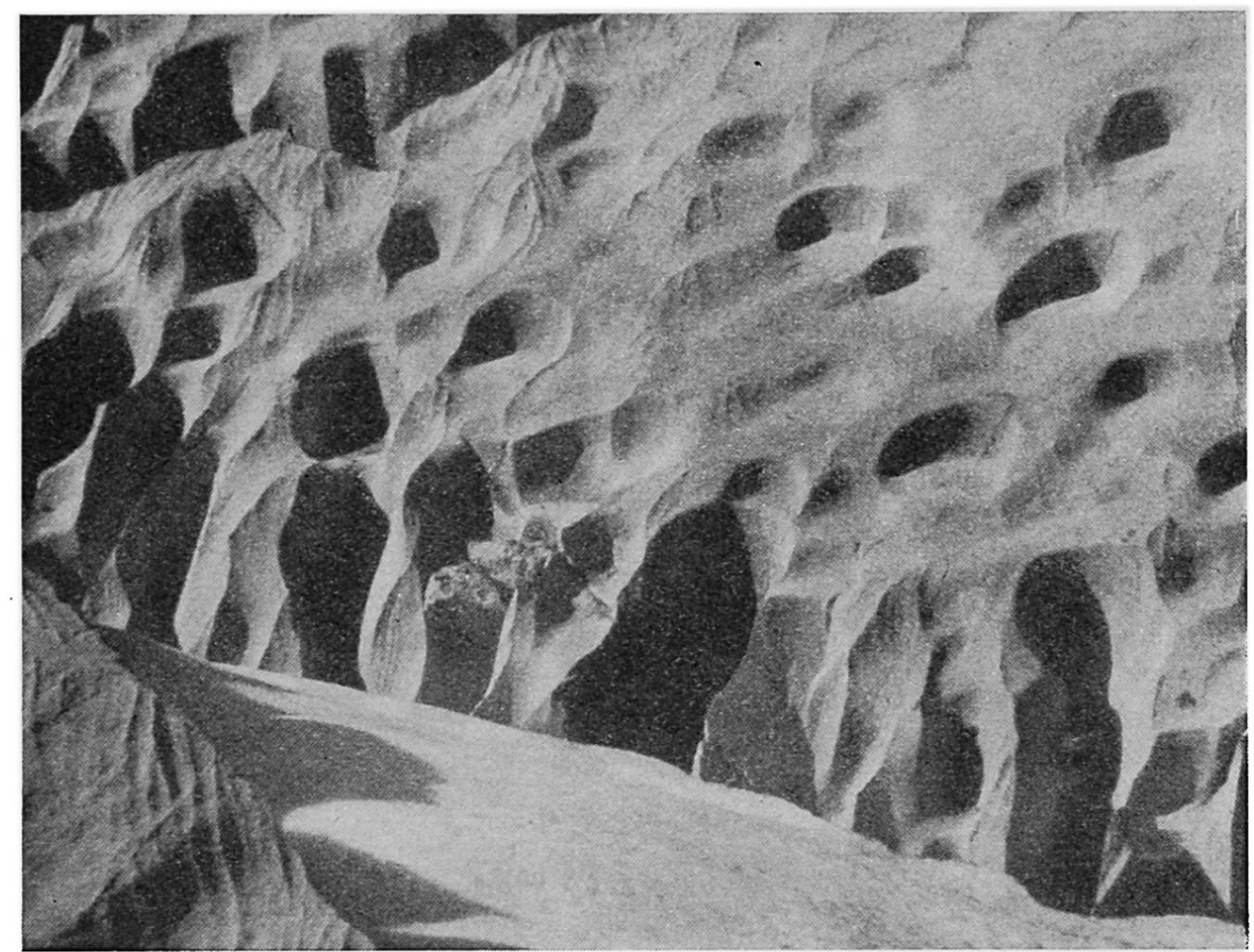

Abb. 4 Westlich Berglikopf (Kinziggebiet), $2130 \mathrm{~m}$, Quintnerkalk. Die Form der Trichterkarren ist umso besser, je länger der Schnee liegen bleibt. Die Kluftkarre ist durch Restschnee (August) noch verdeckt

Auf den wenig geneigten Flächen des Kinzigpaßgebietes haben die Trichterkarren nachweislich ihren Ursprung am Rande der Kluftkarren genommen und beim Rückwärtsschreiten eine dazu parallele Lage erreicht. Die Folge ist die Erniedrigung der ganzen Fläche um $8 \mathrm{~cm}$ für die erste Stufe. Die Formen sind um so typischer ausgebildet, je näher sie einer schattenspendenden Böschung mit ihren Schneeanhäufungen liegen. Es scheint, daß primär die zahlreichen subnivalen Tropfstellen den Anstoß zur Entstehung der Trichterkarren gegeben haben. Jede ursprüngliche Vertiefung der Kalkfläche mußte am Relief der Schneeunterseite zur Tropfstelle werden, die sich jedes Jahr an der gleichen Stelle neu bildet. Die Gefällsversteilung durch Selbstverstärkung erfolgt vorwiegend in der schneelosen Zeit. Erst der Wechsel zwischen der lokalen Tropfstelle des schmelzenden Schnees mit der flächenhaften Benetzung durch Regen vermag die typischen Formen der Trichterkarren zu sčhaffen.

Chemische, vielleicht auch physikalische Unterschiede führen zu Ausgleichsböden, die ungefähr auf gleicher Höhe liegen und dann seitlich verschmelzen. Es bilden sich Bänke, die kaum mehr ihre Enstehung aus Trichterkarren verraten. Dieser Typ - wir haben ihn als Bankkarren bezeichnet ist selten.

Sobald die Wassermenge regelmäßig ein gewisses Maß überschreitet, schließen sich entsprechend gelegene Trichterkarren zu gestuften Rinnenkarren zusammen, in denen sich die Ausgleichsflächen noch lange halten und dadurch einen flachen Karrenboden hervorrufen. Rinnenkarren s. s. und Trichterkarren schließen sich gegenseitig aus. Sobald das Schmelzwasser auch kanalisiert wird, hört die Bildung der Trichterkarren auf, auch dann, wenn die übrigen Bedingungen günstig sind.

Rillenkarren. LeHManN 10 und Rathjens 15 verwenden den Ausdruck Rillenkarren für die in der Literatur häufig als Kannellierungen bezeichneten Formen. Sie sind morphographisch an den regelmäßig angeordneten, durch scharfe Firste getrennten, parallelen, lückenlos sich folgenden, 2 bis $5 \mathrm{~cm}$ breiten und 1 bis $2 \mathrm{~cm}$ tiefen Rinnen mit muldenförmigem Querschnitt leicht von den andern Karrentypen abzutrennen. Sie beginnen am obern Rande steiler Flächen und verschwimmen nach unten plötzlich oder gehen in Regenrinnen über. Ihre Länge hängt vom Ge- 


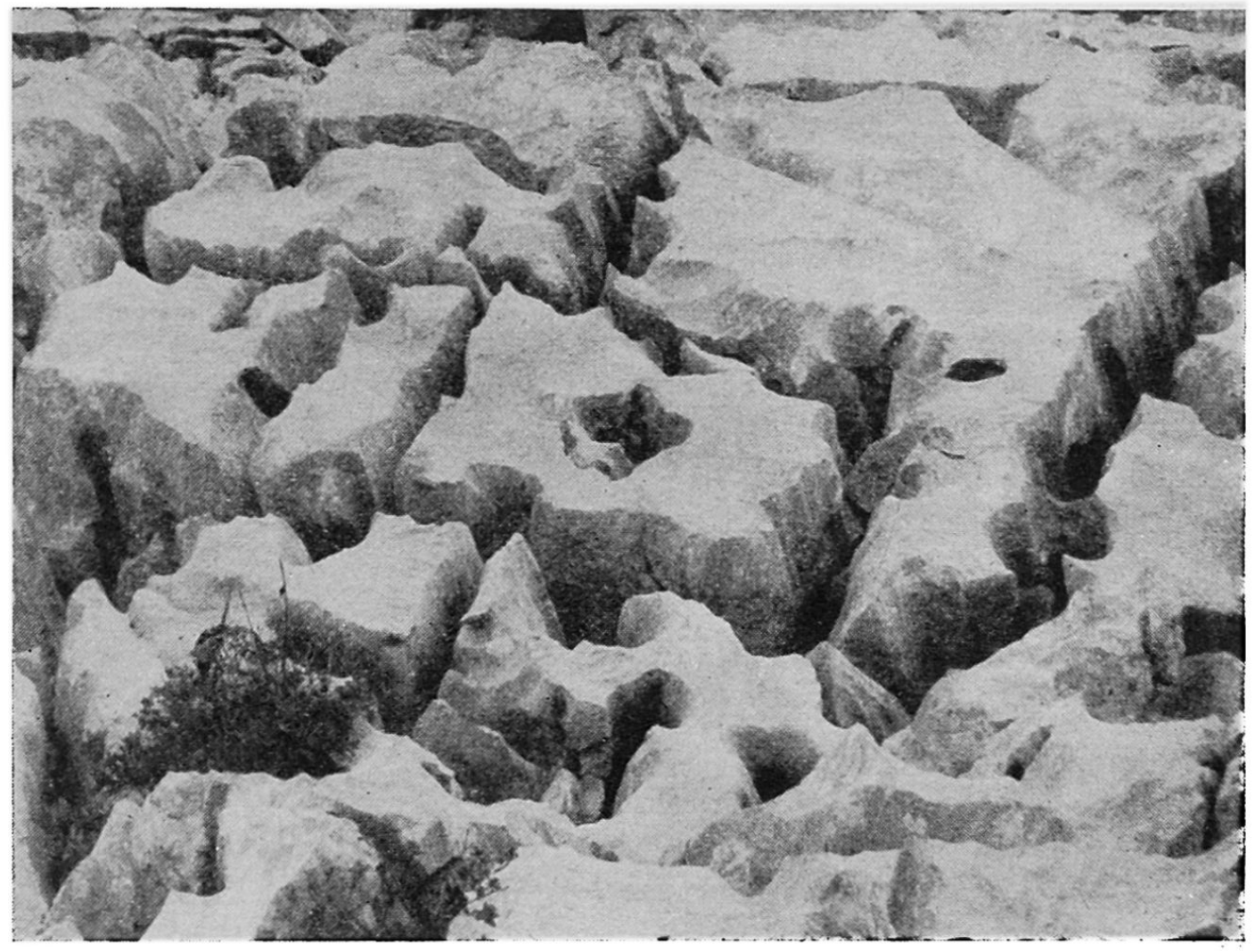

Abb. 5 Schrattenfluh-Hengst, $1950 \mathrm{~m}$, Schrattenkalk. Flachkarren. Die ursprüngliche Fläche ist korrosiv um ein unbekanntes Ausmaß erniedrigt worden und trägt sehr flache Rinnenkarren

fälle ab. Die längsten entstehen an senkrechten Felswänden und übersteigen einen halben Meter.

Die Lage am obern Rande - von dieser Regel konnten wir bisher keine sichere Ausnahme finden - zeigt, daß zur Bildung der Rillenkarren neues, unverbrauchtes Niederschlagswasser von maximaler Lösungskraft notwendig ist. An Stellen, wo aus höheren Teilen Wasser in die Fläche einläuft, sind die Rillenkarren unterbrochen, was die obige Erklärung stützt. Wenn das ablaufende Wasser ein genügend dickes Auffangpolster für die Regentropfen bildet, so daß sie nicht in direkte Berührung mit der Grenzschicht kommen, fehlen die Rillenkarren. Sie sind daher in der Länge infolge der Zunahme des abfließenden Wassers begrenzt.

Morphographisch liegt der Akzent in der großen Regelmäßigkeit, ja Gesetzmäßigkeit in Anordnung und Form. Von den Kräften, die den Lauf des Wassers beeinflussen, ist die Oberflächenspannung die einzige, die im Stande wäre, solche Formen zu erzeugen. Sie kommt aber nur zur Geltung, wenn infolge höheren Gefälles die Druckkomponente des Wassergewichtes klein genug geworden ist. Dann beginnt das Wasser Stränge zu bilden, in deren Stromstrich die Grenzschicht am stärksten angegriffen wird. Zwischen den Strängen läuft nur wenig Wasser, so daß diese Teile sich allmählich als Grate zwischen den Rinnen herausheben. Dadurch wird die Strangbildung unterstützt und fixiert. Die trennenden Grate werden in um so größerem Maße durch die auftreffenden Regentropfen korridiert, je stärker sie hervortreten. Das setzt ihrer Höhe eine Grenze.

Die Rillenkarren sind im allgemeinen Gebilde der höheren Karrenregionen, doch konnten wir mehrfach an großen Blöcken bis zu $800 \mathrm{~m}$ Meereshöhe hinunter solche Formen nachweisen. Auf anstehendem Schrattenkalk findet man inmitten des Nadelwaldes der Bödmeren (Bisistal) auf $1380 \mathrm{~m}$ Höhe fast modellhafte Rillenkarren. Im nordalpinen Klima können sich demnach Rillenkarren bis auf die Talsohle hinunter bilden, wenn neben dem Gefälle die Hauptbedingung der Vegetationslosigkeit erfüllt ist.

Flachkarren (Abb. 5) sind nicht Einzelformen, sondern Formkomplexe. Im Prinzip handelt es sich um eine Kalkfläche, die durch tiefe Kluft- und Rinnenkarren in Einzelteile zerlegt worden ist. Dieser Formkomplex spielt bei Karrenbeschreibungen merkwürdigerweise keine Rolle, obschon er am Landschaftsbilde vorherrschend beteiligt ist. Von besonderer Bedeutung ist er für die Altersbestimmung der Karren. 


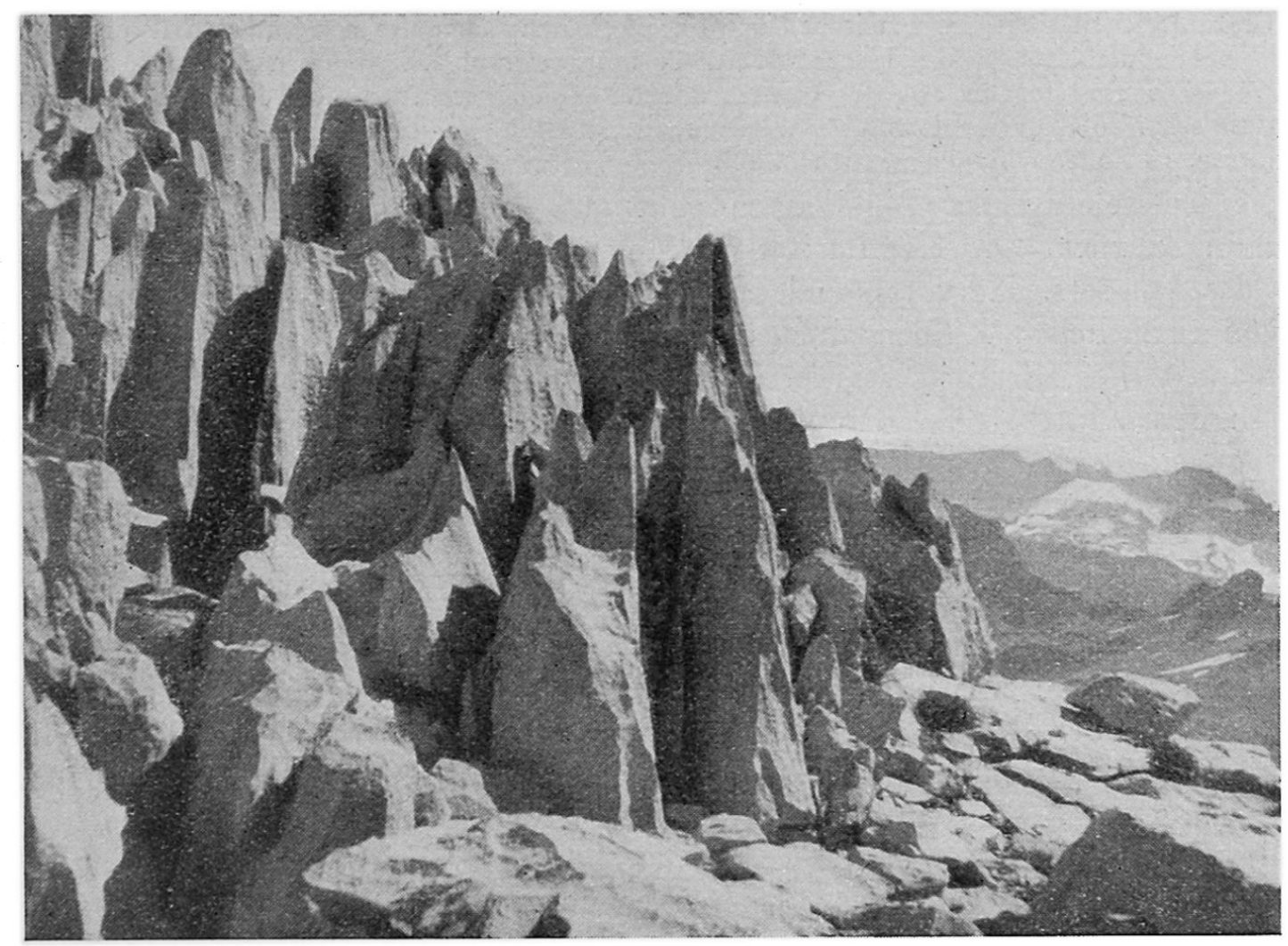

Abb. 6 Misthaufen (Kaiserstockkette), 2230 m, Schrattenkalk. Spitzkarren, an Flachkarren grenzend, zwei bis drei Meter hoch

Die Kalkflächen tragen Rinnenkarren von normaler Breite, aber sehr geringer Tiefe, so daß sie das Bild der Fläche nicht stören. Sie sind unabhängig von den tiefern Karren entstanden und wirken sehr jung.

Spitzkarren (Abb. 6) sind die reifen Formen von Kluft- und Rinnenkarren. Durch Vertiefung und Verbreiterung der Karrenschründe werden die Flächen der Flachkarren aufgezehrt. Übrig bleiben keilartige Zapfen und Firste. Sie bilden eine Gipfelflur, die ein oberes Korrosionsniveau darstellt, dem obern Denudationsniveau entsprechend. Spitzkarren gehören zu den Seltenheiten. Wir fanden sie bisher in schönster Ausbildung auf dem Gipfel des Misthaufens (Kaiserstockkette) bei P. 2268,5 m und etwas weniger schön auf dem Gipfel der Hächlen (Schrattenfluh), P. 2092, 0 alter Wert.

Die T'rümmerkarren bilden, um mit Davis zu sprechen, den Greisenzustand der Karrenentwicklung. In der Zentralschweiz konnten unter normalen Bedingungen keine gefunden werden, da zu deren Entstehung viel zu große Zeiträume notwendig sind. In dünnbankigen Kalken treten sie als Seltenheit auf. Davon sind die durch Spaltenfrost entstandenen Trümmerfelder in schiefrigen Kalken zu unterscheiden, da sie mit der Karrenbildung nichts $\mathrm{zu}$ tun haben.

Rundkarren sind genetisch umstritten. ECKERT 5 erwähnt Karren mit runden Firsten und vermutet die Entstehung der Rundung aus der Humusbedeckung. Lindner 12 dagegen lehnt diese Auffassung ab. Er bezeichnet die runden Karren àls Reifeform.

In der Zentralschweiz liegen die runden Karren vor allen Dingen da, wo eine Humusdecke die Karren überzieht, aber auch neben schroffen Karren an andern Stellen. Wir haben Rundkarren, wie wir sie weiterhin nennen werden, nie an Stellen angetroffen, wo eine frühere Humusbedeckung nicht wahrscheinlich gewesen wäre. Im Gschwend (Bödmerenwald-Bisistal) ragen auf $1400 \mathrm{~m}$ Höhe verkarstete Rundhöcker von Schrattenkalk aus dem Walde, zeigend, daß im Bereiche der Humusbedeckung die Karren rund sind, darüber aber scharf. 
Die zahlreichen Übergänge zwvischen den gewöhnlichen Karrenformen und den Rundkarren verwischen bezeichnenderweise das klare Bild dieser morphographisch so leicht zu definierenden Form keineswegs. Es sind logisch aus der Genese abzuleitende Zivischenformen. Eine Formenreihe verbindet die scharfen Karren mit den Rundkarren, die andere zeigt die neuerliche Zuschärfung nackter Rundkarren. In höheren Lagen werden die entblößten Flächen zuerst durch Rillenkarren angegriffen.

Die Entstehung der Rundkarren geht auf den Ausgleich durch die Humusbedeckung zurück. Die Kapillaritätswirkung der Humusteilchen hemmt den freien Fluß des Wassers. Hervortretende Stellen werden stärker gelöst als tiefere, wenn wir von eigentlichen Rinnen absehen. Dies führt zu einer Abrundung aller Ecken und Kanten. Bei allgemein stärkerem Abtrag ist die Bildung von Karren unterbunden. In den Kluftkarren korrodiert das aggressive Wasser kräftig. Wo ein enges Kluftkarrennetz den Untergrund der Humusdecke zerschneidet, bildet sich ein Gewirr von gerundeten Karrenblöcken.

\section{DAS ALTERSPROBLEM DER KARREN}

In den Flachkarrengebieten lassen sich 2 Rinnenkarrengenerationen unterscheiden (Abb. 5). Die eine bildet auf der Fläche wenige Zentimeter tiefe Gebilde von relativ großer Breite. Diese Rinnenkarren vereinigen sich auf steileren Flächen und bilden dann Rinnen bis zu $20 \mathrm{~cm}$ Tiefe, die irgendwo am Rande der lokalen Platte endigen. Die Zusammengehörigkeit von Fläche und Karre ist eindeutig. In die allgemeine Fläche sind andere Karren, besonders Kluftkarren tief eingeschnitten. Die Rinnenkarren dieser Generation übertreffen die zuerst erwähnten an Größe um ein vielfaches.

Mit Hilfe des meßbaren fehlenden Gesteinsvolumens läßt sich auf Grund der weiter oben berechneten Abtragsmasse die minimale Entstehungsdauer der Hohlform bestimmen. Es fehlt jedoch die Kenntnis der Lage der ursprünglichen Fläche. Wir legen deshalb eine Bezugsfläche als Berührungsebene durch die Spitzenflur. Der so gefundene Wert wird um einen unbekannten Betrag zu klein ausfallen.

Die Spitzkarren sind die älteste erhaltene und berechenbare Form. Ihre Höhe beträgt ca. $300 \mathrm{~cm}$. Es fehlen pro Quadratmeter mindestens $1,5 \mathrm{~m}^{3}$ Fels. Da die Spitzenflur dem obern Korrosionsniveau entspricht, so dürfte der wahre Abtragswert noch höher sein. Unter Zugrundlegung des Abtragsvolumens von $12,5 \mathrm{~cm}^{3}$ pro $\mathrm{m}^{2}$ und Jahrtausend ergibt die Berechnung einen minimalen Zeitbedarf von 120000 Jahren für die Bildung der Spitzkarren. Sie entstanden aus Kluft- und Rinnenkarren, so daß damit deren interglaziales Alter bewiesen ist.

Die Konsequenzen für die Flachkarren sind in. Anbetracht der morphologischen Zusammenhänge Schwerwiegend. Neben den beiden, uns bekannten Spitzkarrenfeldern liegen normale Flachkarren (Abb.6). Der am Rande der Bergkuppe abwandernde Firn hat hier die Kalkbank, deren Zusammenhang durch die tiefgreifenden Karren gelockert war, stückweise abgeschoben. Der obere Teil der spitzkarren verschwand; Flachkarren blieben zurück. Sie sind somit Reliktformen.

An den Flachkarren wurden die Karrenhohlräume möglichst typischer Flächen vermessen. Kluftkarren konnten bis auf eine Ausnahme ganz vermieden werden, ebenso Stellen großer Karrendichte. Im Folgenden sei eine Auswahl besprochen.

Eine Gruppe lag in einer $50 \mathrm{~cm}$ dicken Bank von Quintnerkalk auf der Karrenalp (ca. $1900 \mathrm{~m}$ ). Die Karren waren in etwa $50 \mathrm{~cm}$ Tiefe mit Quintnerkalkbrocken verstopft, doch konnte festgestllt werden, daß die untere Schichtfläche von den Karren durchbrochen worden war. Auf eine Ausräumung wurde verzichtet, jedoch nur der freie Raum in die Berechnung einbezogen. Durchschnittlich fehlten hier pro $\mathrm{m}^{2}$ Fläche $230 \mathrm{dm}^{3}$ Gestein. Seit dem Gschnitzstadium können aber erst 100 bzw. $130 \mathrm{dm}^{8}$ weggeführt worden sein. Die tiefen Rinnenkarren sind also älter. Es fehlen jedoch die Kubaturen der schutterfüllten Karrenräume und der unbekannten Differenz zwizchen Spitzenflur und ursprünglicher Fläche.

Die Herkunft der Trümmer ist nicht gesichert. Es scheint sich um Erratika zu handeln. Sie gleichen nicht restlos dem umgebenden Gestein, doch ist ein sicherer Entscheid schwierig zu treffen. Allfällige Gletscherschrammen wären unter dem Einflusse der Atmosphärilien längst verschwunden. Vom Standpunkt des Geologen aus haben Moränen und Erratiker die größte Beweiskraft.

Eine dicke Kalkbank an der Basis der Zementsteinschichten ergab das Fehlen von $396 \mathrm{dm}^{3}$ Gestein pro $\mathrm{dm}^{2}$, wovon $86 \mathrm{dm}^{3}$ auf Rechnung der jüngeren Generation, die etwa $60 \%$ der Fläche einnimmt, gehen. Die jüngere Generation zeigt damit einen Gesteinsverlust, der in der Größenordnung des Abtrages seit Gschnitz liegt. Die übrigen $310 \mathrm{dm}^{3}$ fallen damit in eine Korrosionsphase, die vor das Gschnitzstadium zu stellen ist und ca. 25000 Jahre umfaßt haben muß. 


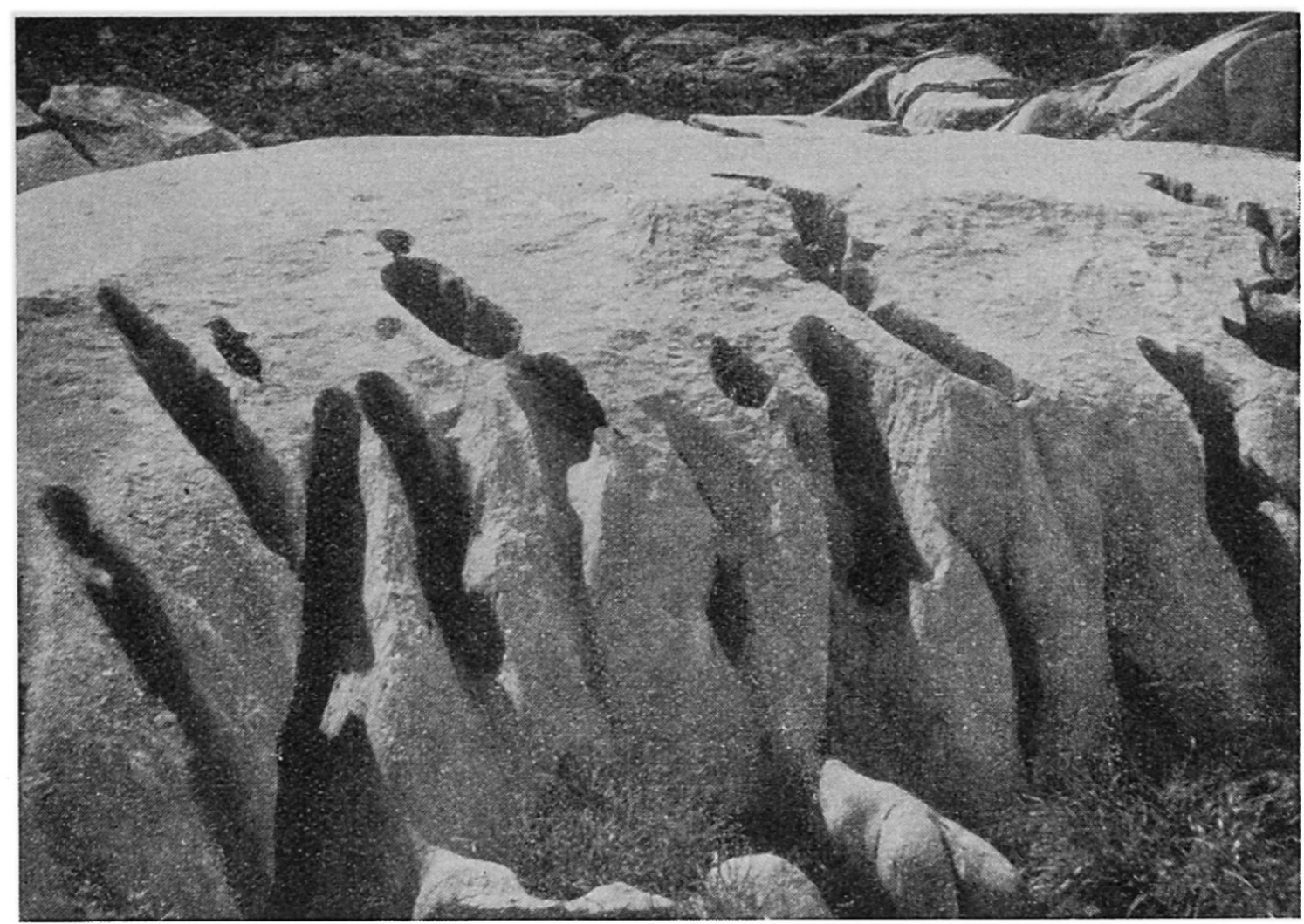

Abb. 7 Zwischen Blümenberg und Kaiserstock, 1800 m, Schrattenkalk. Leeseite einer glazial überschliffenen Kalkbank mit Resten alter Rinnenkarren. Diese müssen älter sein als der Schliff, sind also diluvial

Die Messung, bei welcher die Kluftkarre nicht umgangen werden konnte, ergab einen Karrenhohlraum von $900 \mathrm{dm}^{3} / \mathrm{m}^{2}$.

Firn vermag zwar gut zu erodieren, doch ist der Abtrag auf den weiten Karrenfeldern über das Losbrechen der Spitzkarren hinaus sehr gering. Schichtköpfe und andere Unebenheiten wurden jedoch zu Rundhöckern umgeschliffen. Es ist bezeichnend, daß solche Rundhöcker weniger tiefe Karren der älteren Generation besitzen als das übrige Karrenfeld. In einzelnen Fällen ist durch den glazialen Schliff jede Spur weggeräumt worden. Seither haben sich keine neue Karren von einiger Tiefe eingefressen. Auf der Leeseite sind in der Regel gute Überreste der ältern Generation erhalten geblieben (Abb. 7). Solche morphogenetische Beweise für diluviales Alter der Karren konnten besonders im Schrattenkalk der Kaiserstockkette, der Twärenen und der Schrattenfluh, sowie auf dem Quintnerkalk der Karren- und Glattalp gefunden werden. Die tiefer gelegenen Funde bieten infolge der Einwirkung der Vegetation und der jungen Karrenbildung ein weniger klares Bild.

Bisher galt die Auffassung vom postglazialen Alter der Karren, obschon Heim ${ }^{6}$ diluviale Karren unter Moränen beobachtete und beschrieb und ARBENz ${ }^{1}$ ausdrücklich auf deren Bestehen aufmerksam machte. Wie wir zeigen konnten, gelten die von den beiden Forschern an moränenbedeckten Kalkflächen gemachten Beobachtungen auch für die offenen Karrenfelder. Kluftkarren und tiefe Rinnenkarren sind diluvial, flache Rinnenkarren und Rillenkarren postglazial.

\section{SCHLUSS}

Die vorliegenden Untersuchungen wecken das Bedürfnis nach einer Karrendefinition. EckerT 5 definiert die Karren als eine in verhältnismäßig reinem Kalkstein vorkommende typische Oberfächenerscheinung, die sich in Furchen und dazwischen liegenden Firsten äußert, und deren Entstehung an die Inhomogenitäten 
und an die durch Gebirgsdruck entstandene Klüftungsfähigkeit des Kalksteines i. a. und an die Wirkung der Atmosphärilien und pflanzlichen Organismen im besondern gebunden ist. Diese Definition ist unklar und zu eng, wie Lindner 12 (S. $10 \mathrm{f}$. ) durch Gegenüberstellung seiner eigenen Auffassung zeigt, der u. a. auch Dolomit und mit einiger Reserve Gips in den Kreis seiner Definition zieht.

Wegen der Vielfalt der Formen möchten wir auf die morphographische Definition, die an sich ein Objekt immer unzulänglich erfaßt, verzichten und den Versuch machen, an ihre Stelle die genetische zu setzen.

Karren sind subaerische Korrosionsformen, die auf Karbonatgesteinen und Gips bei flächenhafter Benetzung durch Atmosphärilien und Sprühwasser und durch das dabei ablaufende Wasser unter gelegentlicher Mitwirkung von Organismen entstehen.

Durch diese Definition werden auch Brandungskarren erfaßt, während die Erosionsformen in Schluchten und die Korrosions- und Erosionsformen in Höhlen ausgeschlossen werden. Die karrenartigen Korrosionsformen, die PALMER 14 von den Basalten der Insel Oahu beschreibt, sind auszuscheiden, da nicht Atmosphärilien, sondern das über die Felsen abfließende Grundwasser die Ursache ihrer Entstehung ist, ganz abgesehen davon, daß Basalt nicht in den Kreis verkarrungsfähiger Gesteine einbezogen wurde. Solche Gebilde werden zusammen mit den von Lindner 12 (S. 2) erwähnten auf den Graniten von Brasilien, Südafrika und Seychellen als Pseudokarren bezeichnet.

\section{LITERATUR}

(Von den 59 zur Bearbeitung des Problems verwendeten Werken sind nur jene erwähnt, die bei der Niederschrift der Studie beigezogen werden.) 1. Arbenz, P., Exkursion in die Obwaldner Alpen. Eclogae geol. Helv., Bd. XII, 1912. 2. BœeGLI, A.. Karren. Leben und Umwelt, 4. Jahrg. Heft 3, 1947. 3. BL@ECHLIGER, G., Mikrobiologische Untersuchungen an verwitternden Schrattenkalkfelsen. Diss. ETH, Zürich 1931. 4. DüGgelı, M., Bakteriologische Studien im Karrengebiet der Frutt. Vierteljahresschrift der Natf. Ges. Zürich, Bd. LXXIX, 1934. 5. ECKERT, M., Das Gottesackerplateau. Wiss. Erg. Hefte z. Zeitschr. des DuOeAV, Bd. 1, H. 3, Innsbruck 1902. 6. Heim, Albert, Über die Karrenfelder. Jahrb. SAC 1877/78. 7. JÆKLL, H., Wasser im Stollenbau. Ges. für Militär. Bautechnik, Zürich. 8. KaYser, E., Abriß der Geologie. Bd. II, 1948. 9. v. Klebelsberg, R., Handbuch der Gletscherkunde. 1948. 10. Lehmans, O., Das Tote Gebirge als Hochkarst. Mitt. der geogr. Ges. Wien 1927. 11. Lehmann, O., Die Hydrographie des Karstes. Enzykl. der Erdkunde. 1932. 12. Lindner, H., Das Karrenphänomen. Petr. Mitt., Erg.-Heft 208, Bd. 45, 1930. 13. LürschG, O., Zur Hydrologie der Schweizeralpen. Verh. der Schweiz. Natf. Ges., Thun, 1932. 14. PALMER, H., Karrenbildungen in den Basaltgesteinen der hawaiischen Inseln. Mitt. der geogr. Ges. Wien 1927. 15. RathJENs, C., Geomorphologische Untersuchungen in der Reiteralm und im Lattengebirge. Mitt. der geogr. Ges. München 1939. 16. WeLTEN, M., Pollenanalytische, stratigraphische und geochronologische Untersuchungen aus dem Faulenseemoos bei Spiez. 1944. Veröff. des geobot. Inst. Rübel, Zürich 1944.

\section{L'ORIGINE DES LAPIEZ}

L'auteur examine l'origine et le temps de formation des lapiez sur la base des données chimiques et morphologiques. Il démontre que les lapiez de grande profondeur datent du temps glaciaire, les lapiez peu profonds du temps postglaciaire.

\section{LORIGINE DELLE GANE}

L'autore esamina l'origíne e il tempo di formazione delle gane in base ai datici e morfologici; prova che le gane di grande profondità datano dai tempi glaciale, le gane poco profondi dai tempi postglaciali. 